\title{
REVIEW
}

\section{Axitinib in Metastatic Renal Cell Carcinoma}

Kriti Mittal · Laura S. Wood · Brian I. Rini

To view enhanced content go to www.biologicstherapy-open.com

Received: July 25, 2012 / Published online: October 16, 2012

(C) The Author(s) 2012. This article is published with open access at Springerlink.com

\section{ABSTRACT}

Targeted agents have revolutionized the management of metastatic renal cell carcinoma (RCC). Axitinib, an inhibitor of vascular endothelial growth factor receptor (VEGFR), has been an important addition to currently available therapies for advanced RCC. Its ability to inhibit VEGFRs at nanomolar concentrations distinguishes it as a potent tyrosine kinase inhibitor, with increased selectivity for VEGFR-1, 2, and 3 at clinically applicable concentrations. The phase 3 AXIS trial has established its superiority in prolonging progression-free survival (PFS) in previously treated RCC patients (median PFS 6.7 months for axitinib vs. 4.7 months for sorafenib).

K. Mittal $(\bowtie) \cdot$ L. S. Wood · B. I. Rini

Taussig Cancer Institute, Cleveland Clinic, 9500 Euclid Avenue Desk R 35, Cleveland, Ohio 44195, USA e-mail: mittalk@ccf.org

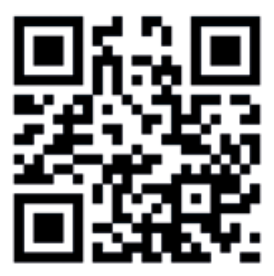

Enhanced content for Biologics in Therapy is available on the journal web site: www.biologicstherapy-open.com
Common toxicities of axitinib include hypertension, diarrhea, nausea, hand-foot syndrome, fatigue, and hypothyroidism. Axitinib-induced diastolic blood pressure elevation may be associated with improved clinical outcome, likely reflecting the "on-target" effect of axitinib. Dose escalation to achieve therapeutic plasma drug levels is of considerable clinical interest. Although axitinib has established efficacy in patients treated with one previous agent, its use in the frontline setting is currently the subject of ongoing research.

Keywords: Advanced renal cell carcinoma; Axitinib; Oncology; Pharmacodynamics; Progression-free survival; Vascular endothelial growth factor receptor

\section{INTRODUCTION}

The treatment of metastatic renal cell carcinoma (mRCC) has historically included immunotherapy, although targeted agents have revolutionized treatment strategies over the last several years. The management of mRCC has evolved rapidly since 2005, when sorafenib, a tyrosine kinase inhibitor (TKI) of vascular endothelial growth factor receptor (VEGFR), 
was approved for advanced RCC following a phase 3 study in patients who had failed to respond to prior systemic therapy $[1,2]$. Since then, newer VEGFR inhibitors, including sunitinib and pazopanib, have been approved in advanced renal cell carcinoma (RCC) in 2006 and 2009, respectively [3-5]. Additionally, bevacizumab, a monoclonal antibody to circulating vascular endothelial growth factor (VEGF), was approved in 2009 for mRCC patients in combination with interferon alpha $[6,7]$. Newer, more biochemically potent VEGF-TKIs, such as axitinib, have expanded the armamentarium of available drugs with targeted action and established efficacy in mRCC.

Angiogenesis and neovascularization are key to proliferation and dissemination of neoplastic cells in many solid tumors. In sporadic RCC, mutations in the Von Hippel-Lindau (VHL) gene lead to decreased degradation of hypoxia-inducing factor (HIF), and subsequent upregulation of VEGF expression [8]. VEGF, a mitogen of endothelial cells, promotes angiogenesis and vascular permeability. Additionally, it also induces expression of plasminogen activators and metalloproteinases; thereby, promoting a prodegradative environment, facilitating cellular migration and invasion [9]. Downstream signaling upon activation of VEGFRs has also been associated with inhibition of apoptosis and decreased production of nitric oxide [10]. Thus, the VHL-HIF pathway is pivotal to the pathobiology of RCC, and has been crucial in the development of targeted agents that include VEGFR inhibitors as well as antibodies to circulating VEGF. Furthermore, mammalian target of rapamycin (mTOR) molecules that are downstream of phosphoinositide 3-kinase and protein kinase B pathways have also demonstrated an increased concentration of HIF-alpha; thus, playing a significant role in angiogenesis [11].
This has expanded available molecular targets and led to the development of mTOR inhibitors, temsirolimus and everolimus, which have demonstrated efficacy in mRCC $[12,13]$.

Since its Food and Drug Administration (FDA) approval in January 2012, the receptor selectivity, potency, high objective response rate (ORR), and tolerability of axitinib have generated considerable interest. The clinical evidence supporting the current role of axitinib in the management of mRCC is reviewed in this article, with a special emphasis on its place in current treatment regimens. In addition, management of drug toxicities and ongoing investigational trials centered on axitinib are detailed.

\section{METHODS}

A PubMed search was conducted using the key terms "axitinib" and "renal cell cancer." Additionally, proceedings from major congresses in North America and Europe over the last 5 years were reviewed for abstracts related to axitinib in RCC.

\section{MECHANISM OF ACTION}

Axitinib is a second-generation, indazolederived molecule that inhibits VEGFR-1, 2, and 3 at therapeutic plasma concentrations; thereby, blocking VEGF-mediated angiogenesis, cellular adhesion, migration, and eventually resulting in cellular apoptosis. Axitinib binds selectively to the adenosine triphosphate (ATP)-binding intracellular domain of VEGFR-1, 2 , and 3 at nanomolar concentrations, stabilizing an inactive confirmation of these kinases and, thus, inhibiting downstream signal transduction [14, 15]. In fact, the half maximal inhibitory concentration (IC50) of axitinib for VEGFR-1, 2, and 3 inhibition is 0.1, 0.2, and $0.1-0.3 \mathrm{nM}$, respectively, distinguishing it as the 
most potent VEGFR-1 inhibitor for the treatment of mRCC [16]. Despite platelet-derived growth factor receptor and C-Kit inhibitory effects observed in vitro, axitinib's potency against non-VEGF receptors is about eight-times weaker and, at clinically achieved plasma levels, VEGF inhibition alone is believed to be responsible for the observed clinical benefits [10]. This selectivity is likely responsible for the potent "on-target" effect and toxicity profile of this agent.

\section{CLINICAL DATA}

In the first phase 1 study of axitinib, 36 patients with advanced solid malignancies were treated with axitinib with total daily dose varying from 10-60 mg [17]. This trial helped define the maximal tolerated dose of axitinib as $5 \mathrm{mg}$ orally twice a day (b.i.d.). Important dose-limiting toxicities included hypertension, diarrhea, and stomatitis. The incidence and severity of hypertension were proportional to drug dosage. Linear pharmacokinetics were observed in the dose range evaluated in this trial. Subsequent phase 1 studies conducted among Japanese and Chinese patients have confirmed similar pharmacokinetics and the adverse-effect profile of axitinib in non-Caucasian subjects $[18,19]$. Another phase 1 study of axitinib in 12 Japanese patients confirmed the tolerability and efficacy of this dose, and demonstrated a significant correlation between axitinib exposure and decline in soluble VEGFR-2 levels [20].

Three independent phase 2 trials subsequently evaluated the role of axitinib in mRCC patients refractory to prior therapy (Table 1) [21-23]. In the initial single-arm phase 2 study published in 2007, 52 mRCC patients with a performance status of Eastern Cooperative Group 0-1, who had previously failed to respond to cytokine therapy, were assigned to receive axitinib starting at $5 \mathrm{mg}$ b.i.d. [21]. The
Table 1 Summary of phase 2 trials of axitinib

\begin{tabular}{llll}
\hline Patients & $\begin{array}{l}\text { ORR } \\
(\%)\end{array}$ & $\begin{array}{l}\text { TTP/PFS } \\
\text { (months) }\end{array}$ & $\begin{array}{l}\text { OS } \\
\text { (months) }\end{array}$ \\
\hline Cytokine refractory [21] & 44.2 & 15.7 & 29.9 \\
Sorafenib refractory [22] & 22.6 & 7.4 & 13.6 \\
Cytokine refractory [23] & 50.0 & 11.0 & NR \\
\hline
\end{tabular}

Table 1 summarizes data from phase 2 axitinib trials, references listed in parenthesis

$N R$ not reported, ORR objective response rate, $O S$ overall survival, $P F S$ progression-free survival, TTP time to progression

primary endpoint was ORR. The median age of the cohort was 59 years, and patients underwent close monitoring for hypertension, proteinuria, and other adverse effects. The ORR was $44.2 \%$ (95\% confidence intervals [CI] 30.5-58.7\%) and after a median follow-up period of 31 months, median overall survival (OS) was 29.9 months (95\% CI 20.3, to not estimable; range 2.4-35.8 months). Another secondary endpoint was time to progression, which was 15.7 months. The 5 -year OS rate of this cytokine-refractory mRCC population was $20.6 \%$, as assessed during longterm follow-up [24]. Also, this longer follow-up did not reveal any new toxicity in the patient cohort [24]. Narrower distribution of peak drug concentration on day 1 of cycle 1 , higher ORR, and better baseline performance status were characteristics significantly associated with 5-year survival.

Subsequently, a second phase 2 study of 62 patients was conducted to evaluate response to axitinib in patients who had received prior VEGF therapy with sorafenib [22]. In this single-arm study, dose escalation was permitted if standard $5 \mathrm{mg}$ b.i.d. dosing was tolerated. Additionally, the protocol allowed for dose reduction or interruption for grade 3-4 (severe, disabling, or life-threatening) nonhematologic toxicities (per National Cancer Institute Common Terminology Criteria for Adverse 
Events [NCI-CTCAE], version 3.0). A total of $53.2 \%$ of patients were able to tolerate dose increases to 7 or $10 \mathrm{mg}$ b.i.d, while $17.7 \%$ of patients required dose reductions to $\leq 5 \mathrm{mg}$ b.i.d. The primary endpoint of ORR was $22.6 \%$ (95\% CI 12.9-35.0\%). With a median follow-up of 22.7 months, median progression-free survival (PFS) was 7.4 months (95\% CI 6.7-11.0 months), and median OS was 13.6 months (95\% CI 8.418.8 months). Interestingly, $80 \%$ of the evaluable patients had some degree of tumor shrinkage.

A phase 2 study of axitinib in Japanese patients who were cytokine refractory has also yielded similar results, with an ORR of $50.0 \%$, and median PFS of 11.0 months, which confirms the previously observed efficacy and tolerability in non-Caucasian populations [23].

\section{Phase 3 AXIS Study}

The AXIS trial was a pivotal, phase 3, randomized controlled study of 723 patients, which compared two targeted agents in mRCC wherein patients were randomized to receive either axitinib $5 \mathrm{mg}$ b.i.d. or sorafenib $400 \mathrm{mg}$ b.i.d. (standard of care) [25]. The primary endpoint of median PFS was 6.7 months for axitinib versus 4.7 months for sorafenib (hazard ratio [HR] for disease progression or death of 0.665 [95\% CI 0.544-0.812], $P<0.0001$; Fig. 1a) [25]. Median PFS for patients who had previously received cytokine therapy was 12.1 months for axitinib and 6.5 months for sorafenib $(\mathrm{HR}=0.464$; 95\% CI 0.318-0.676; $P<0.0001$; Fig. 1b). Among patients previously treated with sunitinib, median PFS was 4.8 months for axitinib and 3.4 months for sorafenib $(\mathrm{HR}=0.741 ; 95 \% \mathrm{CI}$ 0.573-0.958; $P=0.0107$; Fig. 1c). Secondary endpoints included ORR, OS, and safety and tolerability. ORR was $19.4 \%$ (95\% Cl 15.4-23.9\%) versus $9.4 \%$ (95\% CI 6.6-12.9\%) with axitinib and sorafenib, respectively.
The prolonged PFS with both axitinib and sorafenib in sunitinib-refractory cases, although modest, provides evidence favoring lack of crossresistance between different VEGFR inhibitors. These data support the use of sequential VEGFR inhibitors, although optimal sequencing to maximize PFS in mRCC is currently the subject of ongoing research [26-29].

Secondary analysis of the AXIS trial has suggested that of the nearly $54 \%$ of patients (in both sorafenib and axitinib arms) who had received prior sunitinib, those who had received $\geq 9$ months of front-line VEGFR inhibitor tended to have greater PFS (6.3 months vs. 4.5 months for axitinib; 4.6 vs. 2.9 months for sorafenib), although these data are to be regarded as hypothesis-generating given the retrospective nature and small subsets with overlapping CIs [30]. Upcoming trials of sequential therapy may reflect further on this observation.

In the AXIS trial, patient-reported outcomes were assessed by the Functional Assessment of Cancer Therapy Kidney Symptom Index (FKSI) questionnaire, as well as FKSI-Disease Related Symptoms (FKSI-DRS) subscale, at the onset of therapy and then monthly until 28 days after the last drug dose [25]. An analysis of time to deterioration demonstrated $17 \%$ reduction in FKSI $(P=0.014)$ and $16 \%$ reduction in FKSIDRS $(P=0.0203)$ with axitinib compared with sorafenib. These data suggest that the benefit in PFS gained with axitinib is accompanied by a delay in symptoms of advanced RCC in the second-line setting.

\section{PHARMACODYNAMICS AND PHARMACOKINETICS}

Available as $5 \mathrm{mg}$ and $1 \mathrm{mg}$ tablets, axitinib achieves peak plasma concentration in 3 hours after a high-fat, high-calorie meal, and 2 hours after overnight fasting [31]. According to 
(a)

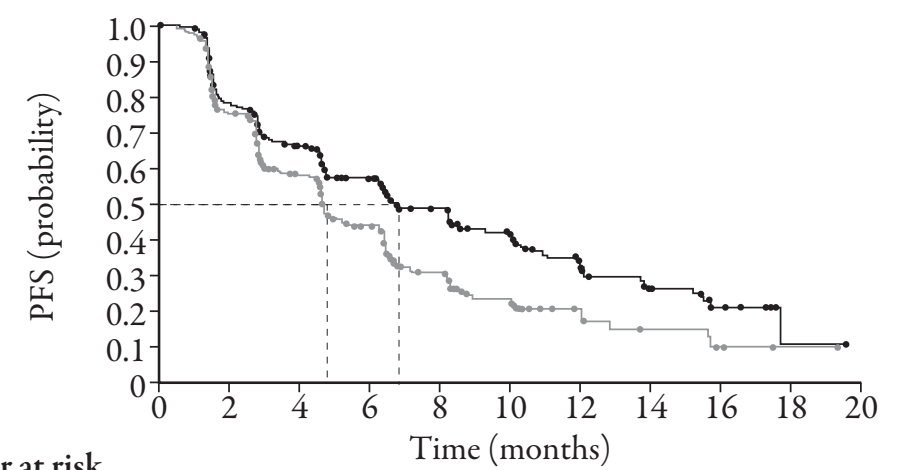

Number at risk

$\begin{array}{llllllllllll}\text { Axitinib } & 361 & 256 & 202 & 145 & 96 & 64 & 38 & 20 & 10 & 1 & 0\end{array}$

$\begin{array}{llllllllllll}\text { Sorafenib } & 362 & 224 & 157 & 100 & 51 & 28 & 12 & 6 & 3 & 1 & 0\end{array}$

(b)

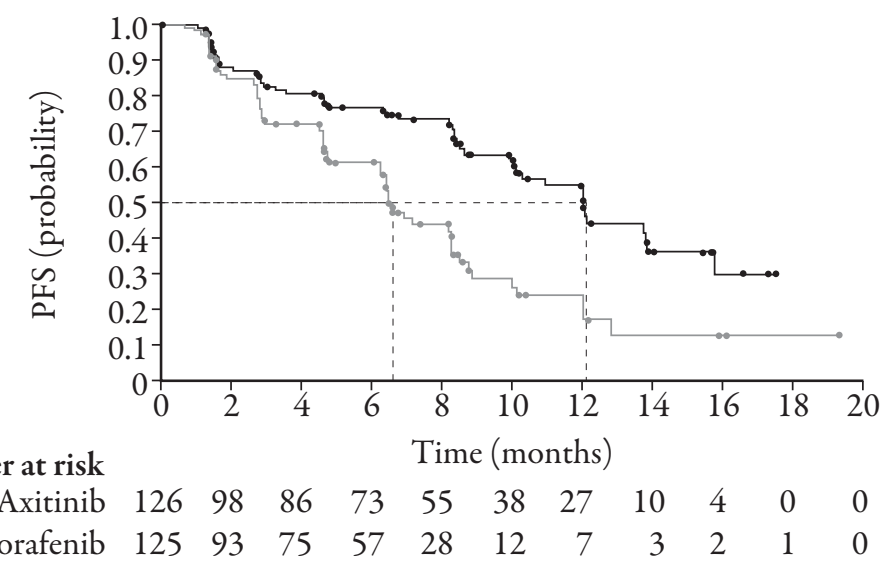

(c)

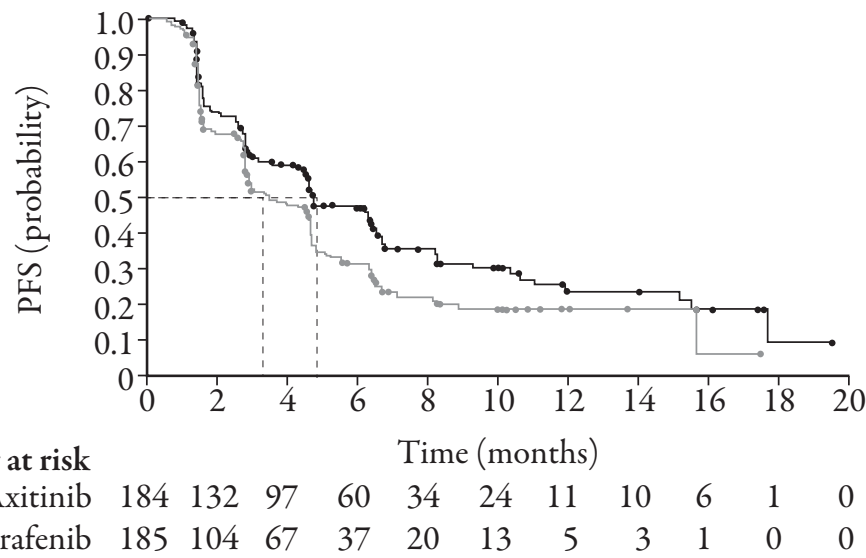

\section{Number at risk}

$\begin{array}{llllllllllll}\text { Axitinib } & 184 & 132 & 97 & 60 & 34 & 24 & 11 & 10 & 6 & 1 & 0\end{array}$

Median PFS (months)

6.7 (95\% CI 6.3-8.6)

$4.7(95 \%$ CI $4.6-5.6)$

$P<0.0001$

Stratified HR $=0.665$

(95\% CI 0.544-0.812)

Median PFS (months)

$12.1(95 \%$ CI $10.1-13.9)$

- Axitinib

$6.5(95 \%$ CI $6.3-8.3)$

$P<0.0001$

Stratified HR $=0.464$

(95\% CI 0.318-0.676)

Median PFS (months)

$4.8(95 \%$ CI $4.5-6.4)$

$3.4(95 \%$ CI $2.8-4.7)$

$P=0.0107$

Stratified HR $=0.741$

(95\% CI 0.573-0.958)
- Axitinib

- Sorafenib
- Axitinib

- Sorafenib

Fig. 1 Kaplan-Meier-estimated median PFS in all patients (a), patients previously treated with cytokine-based regimen (b), and patients previously treated with sunitinib-based regimen (c), who received axitinib or sorafenib as second-line therapy for metastatic renal cell cancer. Reproduced with permission from Rini BI, Escudier B, Tomczak P, et al. Comparative effectiveness of axitinib versus sorafenib in advanced renal cell carcinoma (AXIS): a randomised phase 3 trial. Lancet. 2011;378:1931-9. PFS progression-free survival, $H R$ hazard ratio 
manufacturer's guidelines, it may be taken with or without meals [32]. B.i.d. dosing achieves 1.4-fold greater accumulation compared with once-a-day dosing. With a plasma half-life of 2.5-6.1 hours, steady state is achieved within 2-3 days. The drug is primarily metabolized by cytochrome P450 (CYP) 3A4/5, and to a lesser extent by CYP1A2, CYP2C19, and uridine diphosphate glucuronosyltransferase 1 polypeptide A1 (UGT1A1) [33]. Axitinib is an inhibitor of the efflux transporter P-glycoprotein (P-gp) in vitro, but is not expected to inhibit P-gp at therapeutic plasma concentrations. Axitinib is eliminated by hepatobiliary excretion and phase 1 studies have suggested $<1 \%$ excretion of the drug in urine [17]. Dose modifications are not recommended for patients with mild hepatic impairment (Child-Pugh A) [34]. While axitinib has not been studied in patients with severe hepatic failure (Child-Pugh C), the starting dose should be reduced by $50 \%$ in patients with moderate hepatic failure (Child-Pugh B). The incidence of grade 3-4 transaminitis (defined as aspartate transaminase [AST], alanine aminotransferase [ALT] $>5$-10-times the upper limit of normal) is <1\%; however, baseline and periodic monitoring of AST, ALT, and bilirubin are recommended through the course of treatment for all patients. No starting dose adjustments have been recommended in preexisting mild-to-severe renal impairment, but caution is advised in end-stage renal disease (creatinine clearance $<15 \mathrm{~mL} / \mathrm{min}$ ) [32].

Coadministration of axitinib with strong CYP3A4/5 inhibitors (e.g., grapefruit, ketoconazole, itraconazole, clarithromycin, atazanavir) may increase axitinib plasma concentrations. On the other hand, coadministration with strong CYP3A4/5 inducers (e.g., rifampin, dexamethasone, phenytoin, carbamazepine, rifabutin, and St. John's Wort) may decrease plasma concentrations [32].

\section{Pharmacodynamic Variability}

Investigators recently analyzed single nucleotide polymorphisms (SNPs) in VEGF and related receptors, and their correlation with hypertension and PFS in the phase 3 axitinib AXIS study [35]. Preliminary data suggest that three VEGF-A SNPs revealed potential association between VEGF genotype and PFS. Although these data are preliminary, it is noteworthy that these polymorphisms were not associated with axitinib-related hypertension or diastolic hypertension [35]. Additionally, a meta-analysis of pooled data from 11 axitinib studies has not revealed a significant association linking gene polymorphism for enzymes CYP3A4/5, CYP1A2, CYP2C19, UGT1A1, and P-gp to variations in axitinib pharmacokinetics [36]. Additional investigation into pharmacogenomics as they relate to axitinib efficacy and toxicity is needed.

\section{DOSE ESCALATION}

In the AXIS trial, dose escalation of axitinib was permitted based on physician discretion in patients without hypertension (defined as blood pressure $\geq 150 / 90 \mathrm{mmHg}$ ), absence of toxicities more than grade 2 (by Common Terminology Criteria for Adverse Events [CTCAE] version 3.0), and absence of the use of antihypertensive agents. In patients satisfying these criteria, dose escalation was allowed from 5 to $7 \mathrm{mg}$ b.i.d. after 2 weeks. Additionally, dose escalation was permitted in another 2 weeks to $10 \mathrm{mg}$ b.i.d. in patients who continued to satisfy the above criteria, with additional provision for dose reduction in cases of adverse toxicities and physician discretion used throughout [25].

Results of a secondary analysis did not reveal a significant difference in median PFS among patients who received daily doses of axitinib $\leq 10 \mathrm{mg}$ compared with patients 
receiving higher doses of axitinib, with both groups having better PFS than the sorafenib arm [30]. This result is to be expected on the basis of axitinib pharmacokinetics. That is, patients who tolerated the $5 \mathrm{mg}$ b.i.d. standard starting dose may have subtherapeutic axitinib drug levels. Dose escalation to 7 or $10 \mathrm{mg}$ b.i.d. elevates axitinib drug levels in those patients to above threshold, comparable to patients who do experience hypertension or other toxicity at $5 \mathrm{mg}$ b.i.d. Thus, dose titration with axitinib normalizes blood levels in a subset of patients, allowing them to achieve therapeutic axitinib levels and, thus, an antitumor effect. Given interpatient variability in the ability to achieve therapeutic plasma drug levels, dose escalation of axitinib is critical for optimal administration, as is true for many, if not all oral agents.

Enrollment for a randomized phase 2 study to evaluate efficacy of front-line axitinib with dose titration in 200 treatment-naive patients has been completed [37]. In this study, after 4 weeks of standard dosing ( $5 \mathrm{mg}$ b.i.d.), eligible patients were randomized to a dose titration arm (standard dose plus escalating doses of axitinib, arm A) versus standard dose and escalating doses of placebo (arm B). Eligibility for dose titration was based on absence of hypertension $(>150 / 90 \mathrm{mmHg})$, grade 3 or 4 toxicities, prior dose reduction, and use of more than two antihypertensive agents. Patients not meeting randomization criteria for dose titration continued on standard dosing in a third arm (arm C). Preliminary analysis performed in a subpopulation that had pharmacokinetic assessments on day 15 of cycle 1 indicated that patients with drug exposure above the therapeutic threshold (area under the plasma concentration time curve from $0-12 \mathrm{~h}\left[\mathrm{AUC}_{0-12}\right]$ $\geq 150 \mathrm{ng} / \mathrm{mL}$ ) on day 15 of cycle 1 had a longer median PFS and higher ORR than those with subtherapeutic exposure (median PFS:
13.9 months vs. 8.3 months; ORR: $59 \%$ vs. $48 \%$ ). In a blinded pooled analysis of the entire enrolled population, ORR in arm C was higher $(56.0 \%$; 95\% CI 45.2-66.4\%) compared with arms A+B (40.2\%; 95\% CI 31.0-49.9). These data suggest that response rates and PFS more likely depend on achievement of therapeutic concentration of axitinib, rather than specific drug dosage. This lends support to consideration of dose escalation to improve treatment response, by optimization of plasma drug levels. The unblinding of this study and comparison of patients eligible for dose titration who underwent dose escalation versus patients who did not, will provide more definitive insight into the clinical utility of dose escalation.

\section{Dose Titration}

Currently, the recommended oral starting dose of axitinib is $5 \mathrm{mg}$ b.i.d. Periodic monitoring of blood pressure is recommended. In patients who do not develop dose-limiting toxicity (grade 3 or 4), are normotensive, and are not receiving antihypertensive agents, uptitration to $7 \mathrm{mg}$ b.i.d. 2 weeks after initiation is suggested. Using the same principle, the dose can be increased to a maximum of $10 \mathrm{mg}$ b.i.d. In patients who require dose reduction because of adverse effects, a decrease to $3 \mathrm{mg}$ b.i.d. is recommended. Further dose reduction to $2 \mathrm{mg}$ b.i.d. may be necessary in some cases.

\section{TOXICITIES AND THEIR MANAGEMENT}

Based on results of the AXIS trial, common adverse effects of axitinib that have been observed in over 30\% of patients (all grade toxicities) include diarrhea, hypertension, fatigue, nausea, lack of appetite (anorexia), and dysphonia [25]. Among laboratory abnormalities, 
anemia, hypothyroidism, lymphopenia, and hypocalcemia were commonly seen. Less common side effects included bleeding, thromboembolism, and proteinuria. Table 2 [25] summarizes the various grades of toxicities observed in the phase 3 study. The decreased incidence of myelosuppression, hand-foot syndrome (palmar-plantar erythrodysesthesia),

Table 2 Common treatment-emergent all-causality adverse events in the phase 3 AXIS trial

\begin{tabular}{|c|c|c|c|c|}
\hline \multirow[t]{2}{*}{ Treatment-emergent events } & \multicolumn{2}{|c|}{ Axitinib $(n=359)$} & \multicolumn{2}{|c|}{ Sorafenib $(n=355)$} \\
\hline & All grades & $\geq$ Grade 3 & All grades & $\geq$ Grade 3 \\
\hline \multicolumn{5}{|l|}{ Adverse events } \\
\hline Diarrhea & $197(55)$ & $38(11)$ & $189(53)$ & $26(7)$ \\
\hline Hypertension & $145(40)$ & $56(16)$ & $103(29)$ & $39(11)$ \\
\hline Fatigue & $140(39)$ & $41(11)$ & $112(32)$ & $18(5)$ \\
\hline Decreased appetite & $123(34)$ & $18(5)$ & $101(29)$ & $13(4)$ \\
\hline Nausea & $116(32)$ & $9(3)$ & $77(22)$ & $4(1)$ \\
\hline Dysphonia & $111(31)$ & 0 & $48(14)$ & 0 \\
\hline Palmar-plantar erythrodysesthesia & $98(27)$ & $18(5)$ & $181(51)$ & $57(16)$ \\
\hline Weight decreased & $89(25)$ & $8(2)$ & $74(21)$ & $5(1)$ \\
\hline Vomiting & $85(24)$ & $12(3)$ & $61(17)$ & $3(1)$ \\
\hline Asthenia & $74(21)$ & $19(5)$ & $50(14)$ & $9(3)$ \\
\hline Constipation & $73(20)$ & $4(1)$ & $72(20)$ & $3(1)$ \\
\hline Hypothyroidism & $69(19)$ & $1(<1)$ & $29(8)$ & 0 \\
\hline Cough & $55(15)$ & $3(1)$ & $59(17)$ & $2(1)$ \\
\hline Mucosal inflammation & $55(15)$ & $5(1)$ & $44(12)$ & $2(1)$ \\
\hline Arthralgia & $54(15)$ & $5(1)$ & $39(11)$ & $5(1)$ \\
\hline Stomatitis & $54(15)$ & $5(1)$ & $44(12)$ & $1(<1)$ \\
\hline Rash & $45(13)$ & $1(<1)$ & $112(32)$ & $14(4)$ \\
\hline Alopecia & $14(4)$ & 0 & $115(32)$ & 0 \\
\hline \multicolumn{5}{|l|}{ Laboratory abnormalities $^{a}$} \\
\hline Anemia & $113 / 320(35)$ & $1 / 320(<1)$ & $165 / 316(52)$ & $12 / 316(4)$ \\
\hline Hemoglobin elevation & $31 / 320(10)$ & NA & $3 / 316(1)$ & NA \\
\hline Neutropenia & $19 / 316(6)$ & $2 / 316(1)$ & $26 / 308(8)$ & $2 / 308(1)$ \\
\hline Thrombocytopenia & $48 / 312(15)$ & $1 / 312(<1)$ & $44 / 310(14)$ & 0 \\
\hline Lymphopenia & $106 / 317(33)$ & $10 / 317(3)$ & $111 / 309(36)$ & $11 / 309(4)$ \\
\hline Creatinine elevation & $185 / 336(55)$ & 0 & $131 / 318(41)$ & $1 / 318(<1)$ \\
\hline Hypophosphatemia & $43 / 336(13)$ & $6 / 336(2)$ & $158 / 318(50)$ & $51 / 318(16)$ \\
\hline Hypercalcemia & $19 / 336(6)$ & 0 & $5 / 319(2)$ & 0 \\
\hline Hypocalcemia & $132 / 336(39)$ & $4 / 336(1)$ & $188 / 319(59)$ & $5 / 319(2)$ \\
\hline Lipase elevation & $91 / 338(27)$ & $16 / 338(5)$ & $148 / 319(46)$ & $47 / 319(15)$ \\
\hline
\end{tabular}

Reproduced with permission from Rini BI, Escudier B, Tomczak $P$, et al. Comparative effectiveness of axitinib versus sorafenib in advanced renal cell carcinoma (AXIS): a randomised phase 3 trial. Lancet. 2011;378:1931-9

Data are $n(\%)$

$N A$ not available

${ }^{a}$ Denominator for each laboratory abnormality differed depending on the availability of baseline and at least one on-study test result 
and cutaneous toxicities with axitinib compared with other VEGFR inhibitors is a potential advantage of axitinib.

The most important component of sideeffect management is patient education prior to treatment initiation. The clinician's knowledge of common side effects provides the basis for focused education. Patient education should include necessary information regarding the treatment plan, dosing, and potential drug-drug interactions (Table 3) [38].

Hypertension should be controlled prior to the initiation of therapy. Home blood pressure assessments should be individualized based on the patient's medical history, concomitant medications, and drug therapy. Patients with a

Table 3 Instructions to include in patient information packet for axitinib

- Treatment information regarding axitinib drug formulation and dosing.

- Information regarding possible food and drug interactions, and other precautions to consider with axitinib therapy.

- Possible side effects, prevention, and early management strategies. This should include a discussion regarding which side effects may be prevented or less severe with prophylactic management (i.e., initiation of oral and skin care regimens prior to initiation of treatment).

- Contact information for their nurse, oncologist, and other healthcare team members, including who (and how) to contact when the office is closed. Emphasize the importance of early communication with the nurse/oncologist as side effects develop.

- Specific instructions regarding home monitoring of blood pressure.

- Daily log to record the day, dose, and time of drug administration, along with space to document side effects and blood pressure measurements.

Reproduced with permission from Wood LS, Gornell S, Rini BI. Maximizing clinical outcomes with axitinib therapy in advanced renal cell carcinoma through proactive side-effect management. Comm Oncol. 2012;9:46-55 history of hypertension, cardiac co-morbidities, renal dysfunction, or diabetes should be closely monitored for treatment-induced hypertension [39]. Early awareness of changes in home blood pressure assessments allows the clinician to begin or adjust antihypertensive medications, improve control of hypertension, and minimize the patient's risk of developing hypertension-related toxicity.

Management of axitinib-associated gastrointestinal side effects includes dietary and medication interventions. Information about dietary management of diarrhea should be given to the patient prior to the initiation of therapy, including consumption of bananas and rice, and the avoidance of spicy foods. Pharmacologic interventions include loperamide, diphenoxylate hydrochloride/atropine sulfate, tincture of opium, probiotics, and psyllium fiber supplements [40]. Avoiding caffeinated beverages and increasing fluid intake can decrease the potential for diarrhea, dehydration, and renal dysfunction. Frequent meals, increasing calorie intake, nutritional supplements, and consultation with a nutritionist can minimize the negative impact of anorexia.

Hand-foot syndrome is a class effect of VEGF-TKIs, and requires ongoing assessment and management to control the extent and severity of this adverse event. Assessment of skin condition and noting the presence of calluses or structural abnormalities that increase pressure on bony prominences should be done at baseline, at each clinic visit, and discussed during phone assessments thereafter. Instructions regarding proactive skin care and supportive footwear should be provided at baseline, with ongoing evaluation and adjustment of interventions throughout treatment. The "3C" approach to management of hand-foot syndrome includes controlling calluses, comforting with cushions, and covering with creams, and provides an 
easy-to-remember method for patient education and intervention strategies $[41,42]$.

Treatment modification including interruption and dose modification may be required in spite of effective patient education, proactive strategies, and frequent and ongoing communication to adjust interventions for drug-related side effects. Interventions directed at fatigue include energy conservation, activity enhancement, psychosocial and nutritional interventions, and psychostimulants after ruling out other causes of fatigue [43]. Hypothyroidism is also associated with the increased incidence of fatigue, emphasizing the importance of obtaining baseline and ongoing thyroid function testing $[38,43]$. Hypothyroidism with VEGFR inhibitors may result from inhibition of angiogenesis around the thyroid gland. Thus, periodic monitoring of thyroid function is recommended.

\section{Hypertension as a Biomarker}

Hypertension is a significant adverse effect of axitinib, with reported incidence (all grades included) of approximately 40-50\% [25]. Hypertension as a class effect of VEGF-TKIs may be secondary to reduced production of the vasodilator, nitric oxide, which is a VEGFRmediated phenomenon [44]. Thus, hypertension may be a surrogate marker of "on-target" activity of axitinib. This was revealed in a retrospective study of five, phase 2 trials of single-agent axitinib [45], wherein diastolic hypertension (defined as diastolic blood pressure $\geq 90 \mathrm{mmHg}$ ) was associated with a lower relative risk of disease progression $(\mathrm{HR}=0.76$; 95\% CI $0.54-$ 1.06; $P=0.107]$. Furthermore, in an 8 -week landmark analysis, diastolic hypertension was associated with improved OS (Fig. 2) [45]. In this 8-week analysis, median PFS for the two mRCC studies was noted to be significantly higher in patients with diastolic hypertension: 16.5 versus 6.4 months $(\mathrm{HR}=0.53 ; 95 \% \mathrm{CI}$ $0.31-0.9 ; P=0.019)$. This suggests that diastolic hypertension correlates with drug efficacy [45].

Importantly, treatment of hypertension itself is not expected to affect antitumor activity [15]; thus, axitinib-induced hypertension should be aggressively managed with standard antihypertensives. Additionally, preliminary subset analysis of the phase 2 front-line study of axitinib with dose escalation has suggested that patients with mean increases of diastolic blood

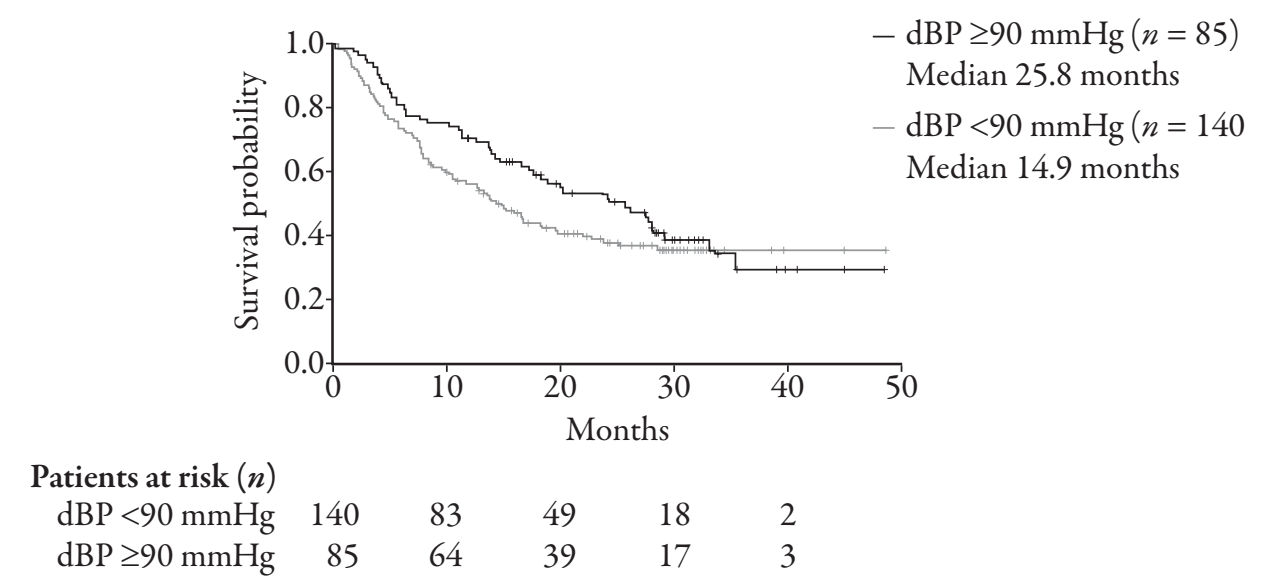

Fig. 2 Overall survival in patients with and without $\mathrm{dBP} \geq 90 \mathrm{mmHg}$ with landmark analysis at 8 weeks. Reproduced with permission from Rini BI, Schiller JH, FruehaufJP, et al. Diastolic blood pressure as a biomarker of axitinib efficacy in solid tumors. Clin Cancer Res. 201 1;17:3841-9. dBP diastolic blood pressure 
pressure at, day 15 of cycle 1 compared with baseline $(\triangle \mathrm{dBP}) \geq 15 \mathrm{mmHg}$ have a higher ORR than $\Delta \mathrm{dBP}<15 \mathrm{mmHg}$ (61\% vs. 53\%) [37].

\section{CONCLUSION}

In conclusion, axitinib has expanded available options for mRCC patients. Selective inhibition of the VEGFR-1, 2, and 3 at clinically applicable concentrations and biochemical potency against VEGFR contribute to its clinical efficacy. Prolonged median PFS is achievable compared with an active comparator in refractory patients, and is currently being evaluated in the front-line setting in mRCC. Analysis of patient-reported outcomes also suggests a delay in symptoms in patients with advanced RCC. Overall, the major dose-limiting adverse effects of axitinib include hypertension, diarrhea, and fatigue. Importantly, hypertension may be a useful biomarker of drug efficacy. Dose escalation is of considerable interest and is undergoing further evaluation in phase 2 studies. Ongoing phase 3 studies are also assessing the role of axitinib in the front-line setting [46], as well as adjuvant treatment in high-risk patients [47].

\section{ACKNOWLEDGMENTS}

Prior to peer review, Pfizer was offered the opportunity to review this paper for scientific accuracy. No writing assistance, other editorial involvement, or financial support was provided by the manufacturer in the production of this manuscript. This article does not necessarily reflect the opinions, policies, or recommendations of Pfizer or any of its employees. Dr. Rini is the guarantor for this article, and takes responsibility for the integrity of the work as a whole.

Conflict of Interest. Dr. Mittal has no personal, commercial, academic, or other financial interests to disclose. Laura Wood has an honorarium for speaker bureau participation from Pfizer. Dr. Rini has consulting and research funding from Pfizer.

Open Access. This article is distributed under the terms of the Creative Commons Attribution Noncommercial License which permits any noncommercial use, distribution, and reproduction in any medium, provided the original author(s) and source are credited.

\section{REFERENCES}

1. Escudier B, Eisen T, Stadler WM, et al. Target Study Group. Sorafenib in advanced clear-cell renal-cell carcinoma. N Engl J Med. 2007;356:125-34.

2. Escudier B, Eisen T, Stadler WM, et al. Sorafenib for treatment of renal cell carcinoma: Final efficacy and safety results of the phase III treatment approaches in renal cancer global evaluation trial. J Clin Oncol. 2009;27:3312-8.

3. Motzer RJ, Hutson TE, Tomczak P, et al. Sunitinib versus interferon alfa in metastatic renal-cell carcinoma. N Engl J Med. 2007;356:115-24.

4. Motzer RJ, Hutson TE, Tomczak P, et al. Overall survival and updated results for sunitinib compared with interferon alfa in patients with metastatic renal cell carcinoma. J Clin Onc. 2009;27:3584-90.

5. Sternberg CN, Davis ID, Mardiak J, et al. Pazopanib in locally advanced or metastatic renal cell carcinoma: results of a randomized phase III trial. J Clin Oncol. 2010;28:1061-8.

6. Escudier B, Pluzanska A, Koralewski P, et al.; AVOREN Trial Investigators. Bevacizumab plus interferon alfa-2a for treatment of metastatic renal cell carcinoma: a randomised, double-blind phase III trial. Lancet. 2007;370:2103-11.

7. Rini BI, Halabi S, Rosenberg JE, et al. Bevacizumab plus interferon alfa compared with interferon alfa monotherapy in patients with metastatic renal cell carcinoma: CALGB 90206. J Clin Oncol. 2008;26:5422-8.

8. Albiges 1, Salem M, Rini B, Escudier B. Vascular endothelial growth factor-targeted therapies in advanced renal cell carcinoma. Hematol Oncol Clin North Am. 2011;25:813-33. 
9. Ferrara N, Davis-Smyth T. The biology of vascular endothelial growth factor. Endocr Rev. 1997;18: 4-25.

10. Hu-Lowe DD, Zou HY, Grazzini ML, et al. Nonclinical antiangiogenesis and antitumor activities of axitinib (AG-013736), an oral, potent and selective inhibitor of vascular endothelial growth factor receptor tyrosine kinases 1, 2, 3. Clin Cancer Res. 2008;14:7272-83.

11. Oudard S, Elaidi RT. Sequential therapy with targeted agents in patients with advanced renal cell carcinoma: Optimizing patient benefit. Cancer Treat Rev. 2012 [Epub ahead of print].

12. Hudes G, Carducci M, Tomczak P, et al. Global ARCC trial. Temsirolimus, interferon alfa, or both for advanced renal-cell carcinoma. N Engl J Med. 2007;356:2271-81.

13. Motzer RJ, Escudier B, Oudard S, et al. RECORD-1 Study Group. Efficacy of everolimus in advanced renal cell carcinoma: a double-blind, randomised, placebo-controlled phase III trial. Lancet. 2008;372:449-56.

14. Kelly RJ, Rixe O. Axitinib - a selective inhibitor of the vascular endothelial growth factor (VEGF) receptor. Targ Oncol. 2009;4:297-305.

15. Patson B, Cohen RB, Olszanski AJ. Pharmacokinetic evaluation of axitinib. Expert Opin Drug Metab Toxicol. 2012;8:259-70.

16. Escudier B, Gore M. Axitinib for the management of metastatic renal cell carcinoma. Drugs R D. 2011;11:113-26.

17. Rugo HS, Herbst RS, Liu G, et al. Phase I trial of the oral antiangiogenesis agent AG-013736 in patients with advanced solid tumors: pharmacokinetic and clinical results. J Clin Oncol. 2005;23:5474-83.

18. Fujiwara Y, Kiyoti N, Chayahara N, et al. Management of axitinib (AG-013736)-induced fatigue and thyroid dysfunction, and predictive biomarkers of axitinib exposure: results from phase I studies in Japanese patients. Invest New Drugs. 2012;30:1055-64.

19. Chen Y, Jiang J, Zhang J, et al. A phase I study to evaluate the pharmacokinetics of axitinib (AG13736) in healthy Chinese volunteers. Int J Clin Pharmacol Ther. 2011;49:679-87.

20. Mukohara T, Nakajima $H$, Mukai $H$, et al. Effect of axitinib (AG-013736) on fatigue, thyroidstimulating hormone, and biomarkers: a phase I study in Japanese patients. Cancer Sci. 2010;101:963-8.
21. Rixe O, Bukowski RM, Michaelson MD, et al. Axitinib treatment in patients with cytokinerefractory metastatic renal-cell cancer: a phase II study. Lancet Oncol. 2007;8:975-84.

22. Rini BI, Wilding G, Hudes G, et al. Phase II study of axitinib in sorafenib-refractory metastatic renal cell carcinoma. J Clin Oncol. 2009;27:4462-8.

23. Tomita $Y$, Uemura $H$, Fujimoto $H$, et al. Japan Axitinib Phase II Study Group. Key predictive factors of axitinib (AG-013736)-induced proteinuria and efficacy: A phase II study in Japanese patients with cytokine-refractory metastatic renal cell carcinoma. Eur J Cancer. 2011;47:2592-602.

24. Motzer RJ, Rouge TD, Harzstark AL, et al. Axitinib second line therapy for metastatic renal cell carcinoma: five year overall survival data from a phase II trial [abstract]. J Clin Oncol. 2011;29(Suppl.). Abstract 4547.

25. Rini BI, Escudier B, Tomczak P, et al. Comparative effectiveness of axitinib versus sorafenib in advanced renal cell carcinoma (AXIS): a randomised phase 3 trial. Lancet. 2011;378: 1931-9.

26. Michel MS, Vervenne W, Goebell PJ, et al. Phase III randomized sequential open-label study to evaluate efficacy and safety of sorafenib (SO) followed by sunitinib (SU) versus sunitinib followed by sorafenib in patients with advanced/ metastatic renal cell carcinoma without prior systemic therapy (SWITCH Study): Safety interim analysis results [abstract]. J Clin Oncol. 2012;30(Suppl.). Abstract 4539.

27. ClinicalTrials.gov NCT01613846. Phase III sequential open-label study to evaluate the efficacy and safety of sorafenib followed by pazopanib versus pazopanib followed by sorafenib in the treatment of advanced/metastatic renal cell carcinoma (SWITCH-II). Available at: clinicaltrials. gov/ct2/show/NCT01613846?term=\%22renal+carc inoma $\% 22+$ and $+\% 22$ sequential $\% 22 \&$ phase $=2 \&$ ra $\mathrm{nk}=2$. Accessed Sep 142012.

28. ClinicalTrials.gov NCT01481870. Comparison of sequential therapies with sunitinib and sorafenib in advanced renal cell carcinoma (CROSS-J-RCC). Available at: http://clinicaltrials.gov/ct2/show/NC T01481870?term $=\% 22$ renal $\% 22+$ and $+\% 22$ sequen tial\%22\&phase=2\&rank=3. Accessed Sep 142012.

29. ClinicalTrials.gov NCT00732914. Sequential study to treat renal cell carcinoma. Available at http:// clinicaltrials.gov/ct2/show/NCT00732914?term $=\%$ 22renal\%22+and+\%22sequential\%22\&phase=2\&ra nk=2. Accessed: Sep 142012. 
30. Rini BI, Escudier B Michaelson MD. Phase III AXIS trial for second-line metastatic renal cell carcinoma: Effect of prior first-line treatment duration and axitinib dose titration on axitinib efficacy [abstract]. Presented at: ASCO 2012 Genitourinary Cancers Symposium. J Clin Oncol. 2012;30(Suppl. 5). Abstract 354.

31. Pithavala YK, Chen Y, Toh M, et al. Evaluation of the effect of food on the pharmacokinetics of axitinib in healthy volunteers. Cancer Chemother Pharmacol. 2012;70:103-12.

32. Inlyta $^{\circledR}$ (axitinib) [package insert]. New York, NY: Pfizer Labs; 2012.

33. Pithavala YK, Tortorici M, Toh M, et al. Effect of rifampin on the pharmacokinetics of axitinib (AG-013736) in Japanese and Caucasian healthy volunteers. Cancer Chemother Pharmacol. 2010;65:563-70.

34. Verbeeck RK. Pharmacokinetics and dosage adjustment in patients with hepatic dysfunction. Eur J Clin Pharmacol. 2008;64:1147-61.

35. Escudier B, Rini BI, Hutson TE, et al. Updated results of the phase 3 AXIS trial: axitinib versus sorafenib as second line therapy for metastatic renal cell carcinoma [abstract]. Presented at: $27^{\text {th }}$ Annual Congress of European Association of Urology; February 24-28, 2012; Paris, France.

36. Brennan M, Williams JA, Chen $\mathrm{Y}$ et al. Metaanalysis of contribution of genetic polymorphisms in drug-metabolizing enzymes or transporters to axitinib pharmacokinetics. Eur J Clin Pharmacol. 2012;68:645-55.

37. Rini BI, Grunwald V, Fishman MN, et al. Axitinib for first-line metastatic RCC: Overall efficacy and pharmacokinetic analyses from a randomized phase II study. Symposium presented at: ASCO Annual Meeting; June 1-5, 2012; Chicago, IL, USA.

38. Wood LS, Gornell S, Rini BI. Maximizing clinical outcomes with axitinib therapy in advanced renal cell carcinoma through proactive side-effect management. Comm Oncol. 2012;9:46-55.
39. Maitland MI, Bakris GI, Black HR, et al. Initial assessment, surveillance, and management of blood pressure in patients receiving vascular endothelial growth factor signaling pathway inhibitors. J Nat Cancer Inst. 2010;102:596-604.

40. Muehlbauer PM, Thorpe D, Davis A, Drabot R, Rawlings BI, Kiker E. Putting evidence into practice: Evidence-based interventions to prevent, manage, and treat chemotherapy and radiotherapy-induced diarrhea. Clin J Oncol Nsg. 2009;13:336-41.

41. Wood LS, Lemont H, Jatoi A, et al. Practical considerations in the management of hand-foot skin reaction caused by multikinase inhibitors. Comm Oncol. 2010;7:23-9.

42. Lacouture ME, Wu S, Robert C, et al. Evolving strategies for the management of hand-foot skin reactions associated with the multitargeted kinase inhibitors sorafenib and sunitinib. Oncologist. 2008:13:1001-11.

43. National Comprehensive Cancer Network. NCCN clinical practice guidelines in oncology: CancerRelated Fatigue v.1.2012 [online]. Available at: http://www.nccn.org/professionals/physician_gls/ pdf/fatigue.pdf. Accessed Jun 292012.

44. Bhargava P. VEGF kinase inhibitors: how do they cause hypertension? Am J Physiol Regul Integr Comp Physiol. 2009;297:R1-5.

45. Rini BI, Schiller JH, Fruehauf JP, et al. Diastolic blood pressure as a biomarker of axitinib efficacy in solid tumors. Clin Cancer Res. 2011;17:3841-9.

46. ClinicalTrials.gov NCT00920816. Axitinib (AG013736) for the treatment of metastatic renal cell cancer. Available at: http://clinicaltrials.gov/ct2/ show/NCT00920816?term=axitinib+and+\%22rena $1+$ carcinoma $\% 22 \&$ phase $=2 \&$ rank $=2$. Accessed Sep 142012.

47. ClinicalTrials.gov NCT01599754. Adjuvant axitinib therapy of renal cell cancer in high risk patients (ATLAS). Available at: http://clinicaltrials.gov/ct2/ show/NCT01599754?term=axitinib+and+\%22rena $1+$ carcinoma $\% 22 \&$ phase $=2 \&$ rank $=1$. Accessed Sep 142012. 